\section{Adopt, adapt, improve}

\section{Derek Richards}

Editor, Evidence-based Dentistry
In this issue we take a look at a recent guidance document from the European Association of Paediatric Document (see page 35). Developing high quality evidence-based guidance documents is a time-consuming and costly process and resources are limited even in the most developed countries, and there seems to be an evergrowing demand for guidelines.

A recent article by Clarkson and Farnham reprinted in three paediatric journals ${ }^{1-3}$ put forward one potential approach for addressing this problem in paediatric dentistry as least. This was the establishing of an international collaborative working to 'help reduce duplication of effort and provide a framework for producing guidelines that are collectively agreed and endorsed.' This type of approach should also help reduce the inconsistencies that exist in guidance documents despite a common evidence-base. This route will not be without its challenges, which exist on a number of levels.

As an indication of the amount of duplication, the Guidelines International Network (http://www.g-i-n.net/) lists 15 guidelines relevant to caries while there are 325 listed on the TRIP database (https://www.tripdatabase.com/). Reducing these levels of duplication should reduce costs and inconsistencies.

The recent agreement at the International Association of Paediatric Dentistry (IAPD) Conference that IAPD was an the appropriate organisation to host a repository for internationally endorsed guidelines and act as a communication hub for paediatric guidelines is an important first step for what is an aspirational development of international guideline development. It will be interesting to see if other dental specialties will follow the international paediatric dentist community and make steps towards international development.

While international cooperation and development will be more of a medium to long-term goal another more cost-effective approach to guideline development is the process of adaption. Between 2006 and 2010 an international collaboration of researchers, guideline developers and guideline implementers began promoting the development and use of clinical practice guidelines through the adaptation of existing guidelines. This group, the ADAPTE Collaboration, developed the ADAPTE Manual and Resource Toolkit and its evaluation. The Guidelines International Network (G-I-N) is now continuing the work in this area and further details and ADAPTE resources are available on the G-I-N website (http://www.g-i-n.net/working-groups/adaptation/ introduction-g-i-n-adaptation-wg)

While adapting existing guidance and developing international guidance in the future are very welcome developments, the quality of guidelines also depends on the quality of the primary evidence and there is also much to be done there to ensure that high quality primary research is conducted, as all too many of the systematic reviews that are conducted are still finding that the available evidence is of low or limited quality to make informed decisions. To a greater or lesser extent the quality of guidelines can only ever be as definitive as the underlying evidence.

Adopt, adapt and improve is a maxim of the Round Table (http:// www.rtinternational.org/about-us/origins/) formed in England in 1927.

The maxim derives from a speech made in 1927 by the then Prince of Wales, who urged young business and professional men to 'ADOPT methods that have proved so sound in the past, ADAPT them to the changing needs of the times and wherever possible, IMPROVE them'.

So while we may adopt and adapt our guideline development processes there is still so much to improve.

1. Clarkson J, Farnham J. Is it time to share paediatric guideline development? Eur Arch Paediatr Dent 2016; 17: 141-142. doi: 10.1007/s40368-016-0225-0. PubMed PMID: 26924433.

2. Clarkson J, Farnham J. Is It Time to Share Pediatric Guideline Development? Pediat Dent 2016; 38: 10. PubMed PMID: 26892207.

3. Clarkson I, Farnham I; IAPD guideline development forum. Editorial: is it time to share paediatric guideline development? Int J Paediatr Dent 2016; 26: 1-2. doi: 10.1111/ ipd.12217. PubMed PMID: 26768344.

Evidence-Based Dentistry (2016) 17, 33. doi:10.1038/sj.ebd.6401161 